\title{
CORRESPONDENCE.
}

\section{ON THE GENERAL EXPRESSION FOR THE FORCE OF MORTALITY.}

To the Elditor of the Journal of the Institute of Actuaries.

Sin, - The general expression for the value of the force of mortality given by you, but not demonstrated, in a foot-note to Mr. Sheppard's communication in the last number of the Journal (J.I.A., xxxii, 295), appears to me to be of sufficient importance to call for a formal proof, and I therefore venture to communicate the following.

Let the value of $l_{x}$ be expressed, by means of Lagrange's interpolation formula, in terms of $l_{-n} \ldots l_{0} \ldots l_{+n}$; we shall have

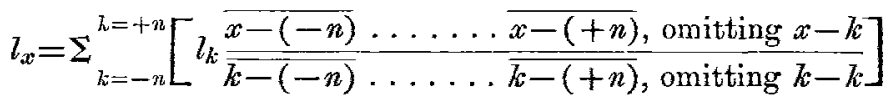

The general term, whether $k$ be,+ 0 or - , may be put into the form

$$
\frac{\overline{x+n} \ldots \ldots \overline{x-n}}{x-\bar{k}}(-1)^{n-k}
$$

whence

$$
\begin{aligned}
& \frac{l_{0}-l_{x}}{x}=\frac{l_{0}}{x}-\Sigma_{k=-n}^{k=+n}\left[l_{k}^{\frac{\overline{x+n} \cdots \cdots \overline{x-n}}{x \overline{x-k}}}(-1)^{n-k}\right]
\end{aligned}
$$

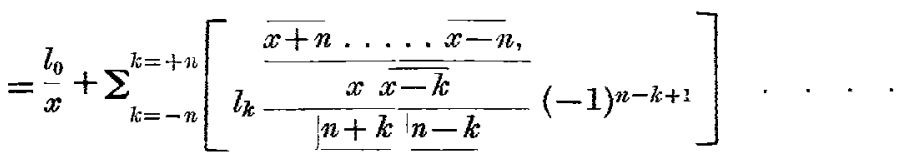


1896.] On the General Expression for the Force of Mortality. 391 Put $x=0$; then $\frac{l_{0}-l_{x}}{x}=-\frac{d}{d_{x}} l_{0}=l_{0} \times \mu_{0}$, and for all values of $k$, except zero, the general term in the expression (a) becomes

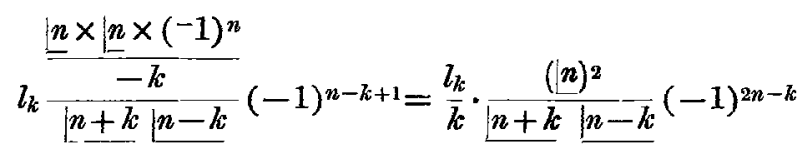

The term involving $l_{v}$ becomes, by adding the two coefficients which appear in (a),

$$
\frac{l_{0}}{n \underline{n}[n} \times L t_{x=0}\left[\frac{\overline{x+n} \ldots \overline{x+1} \cdot \overline{x-1} \ldots \ldots \overline{x-n}(-1)^{n+1}+(\underline{n})^{2}}{x}\right]
$$

which takes the form $\frac{0}{0}$, but it may be shown that when $x=0$ the term vanishes $\left({ }^{*}\right)$. We therefore bave

$$
\mu_{0}=\Sigma \frac{l_{k}}{k l_{0}} \cdot \frac{(\mid n)^{2}}{n+k \mid n-k}(-1)^{2 n-k}, \text { omitting } l_{0} .
$$

Now we have, since any even power of $(-1)$ is equal to unity

$$
\frac{(-1)^{2 n-k}}{k}=-\frac{(-1)^{2 n-(-k)}}{-k}
$$

i.e., the coefficient of $l_{+k}$ is the same as that of $l_{-k}$ with the sign changed; hence the terms may be arranged in pairs. It is also evident that the signs are alternately + and - , and we shall have finally

$$
\begin{aligned}
& \begin{array}{r}
\mu_{0}=\frac{l_{-1}-l_{+1}}{l_{0}} \frac{(\underline{n})^{2}}{\frac{n+1}{\underline{n-1}}}-\frac{l_{-2}-l_{+2}}{2 l_{0}} \frac{\left(\underline{n)^{2}}\right.}{\underline{n+2} \underline{\underline{n-2}}}+. \\
\quad \text { (*) } \overline{x+n} \ldots \overline{x+1} \overline{x-1} \ldots \overline{x-n}(-1)^{n+1}
\end{array} \\
& =\overline{x^{2}-n^{2}} \overline{x^{2}-\overline{n-1}} \ldots \overline{x^{2}-1^{2}}(-1)^{n+1} \\
& =\overline{x^{3}-x^{2}} \overline{n-1^{2}-x^{2}} \ldots \ldots \overline{1^{2}-x^{2}} \times(-1) \\
& =-\left[(\mid n)^{2}+\text { terms involving } x^{2}, \& c .\right] \text {. }
\end{aligned}
$$

Thus, when $x=0$ the fraction of which we require the limiting value is in the form $\frac{\text { Terms involving } x^{2} \text {, \&ce. }}{x}$, and the limiting value is therefore zero. 
In Mr. Sheppard's formulæ the numerical coefficient of $\frac{l_{-k}-l_{+k}}{l_{0}}$ is given in the form

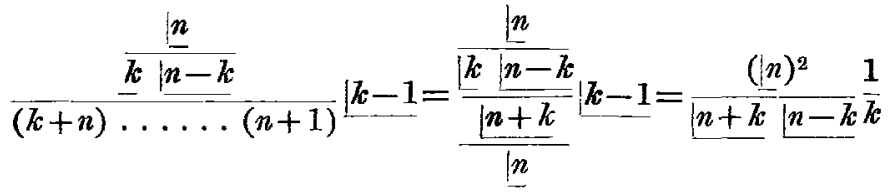

as given above.

The formula having been obtained by the use of $2 n+1$ values of $l$ will be correct to $(2 n)$ th differences.

\section{I am, Sir,}

Your obedient servant, GEORGE J. LIDSTONE.

Bartholomev Lane, E.C., February 1896. 